\title{
Complete esophageal obstruction: an ususual complication of esophageal variceal ligation
}

A 65-year-old woman with cirrhosis presented having suffered from acute chest discomfort and difficulty swallowing for 1 day. Two weeks previously, she had undergone esophagogastroduodenoscopy (EGD) with endoscopic variceal ligation (EVL) for bleeding esophageal varices. Repeat EVL was done on the day before the present admission for continued variceal eradication. After the procedure, the patient developed acute retrosternal discomfort and inability to tolerate oral intake, which worsened with observation.

Gastrografin swallow on admission revealed complete esophageal obstruction at the level of the carina ( $\bullet$ Fig. 1).

Repeat EGD showed a necrotic, completely obstructing mass in mid esophagus which could not be traversed even with a slim endoscope. Three bands were noted in the periphery of the obstructive mass (๑ Fig. 2).

No intervention was performed due to the risk of perforation and the patient was put on total parental nutrition. The following week, she experienced gradual improvement of the dysphagia. Eventually, she was able to tolerate a full diet. An upper gastrointestinal series 2 weeks later showed complete resolution of the esophageal obstruction ( $\mathbf{F i g . 3}$ ).

Variceal bleeding is a major complication occurring in $30 \%-60 \%$ of patients with cirrhosis $[1,2]$. The associated mortality is as high as $20 \%$ within 6 weeks after the initial bleeding, and $60 \%$ of untreated patients experience recurrent bleeding within a year [1]. EVL is the therapy of choice for primary bleeding prophylaxis and postbleeding management $[3,4]$. The procedure is relatively safe, with very few reported complications; these include band site ulceration, bleeding, infection, transient dysphagia, and esophageal perforation caused by trauma [5].

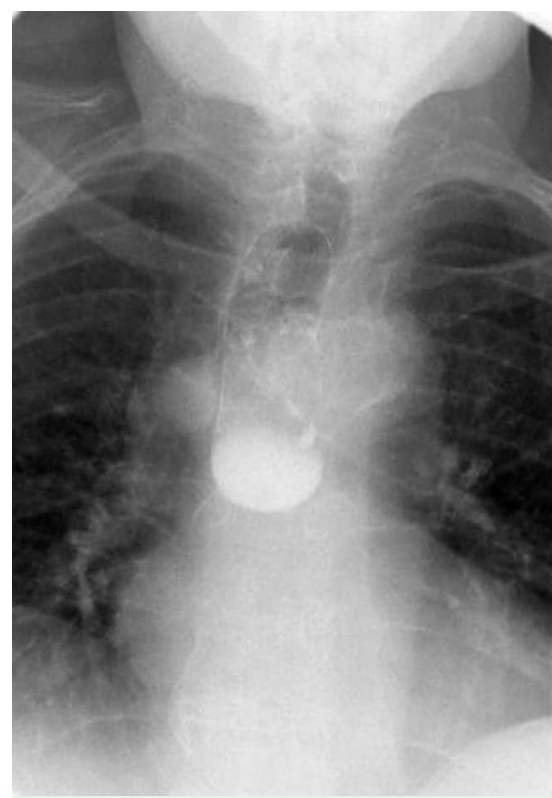

Fig. 1 Gastrografin swallow study showing complete obstruction to the passage of contrast through the esophageal lumen.

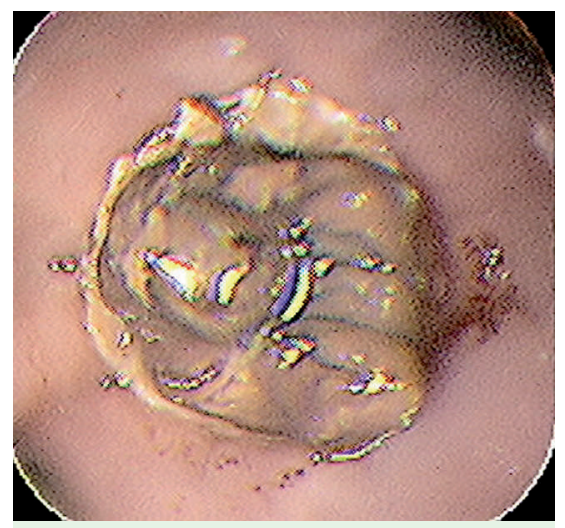

Fig. 2 Endoscopic view of complete obstruction of the esophagus.

Although transient dysphagia can occur following EVL, complete esophageal obstruction is rare. Our patient developed

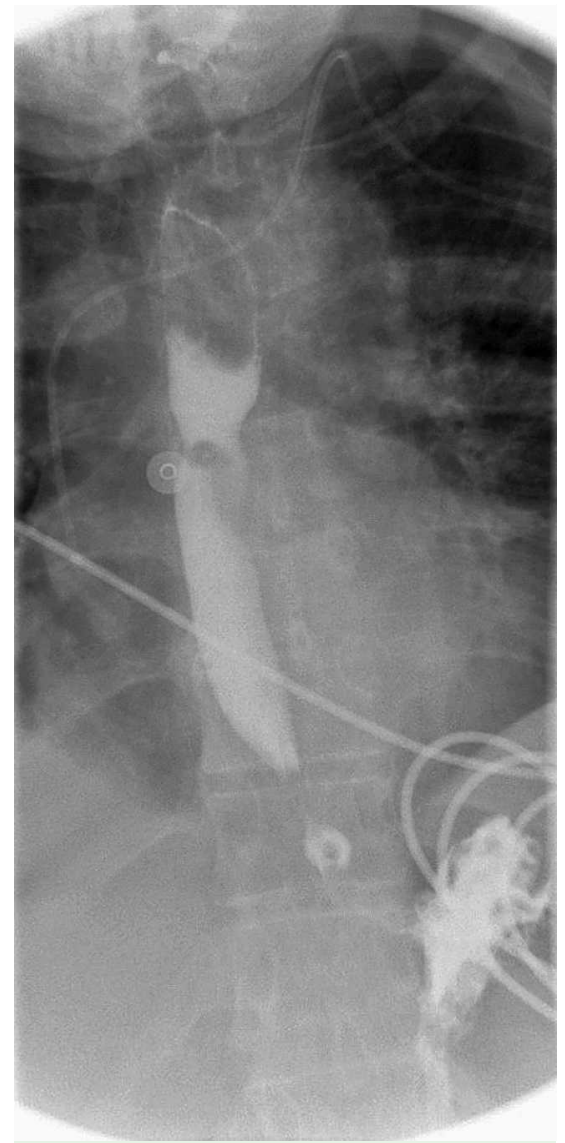

Fig. 3 Resolution of esophageal obstruction with conservative treatment.

complete esophageal obstruction after EVL with significant dysphagia, and the follow-up examination demonstrated esophageal obstruction at the EVL site.

This obstruction occurred due to luminal compromise secondary to tissue edema and necrosis at the banding site. To avoid this, we recommend that EVLs should be applied in a spiral fashion, especially in patients needing multiple sessions, so as to avoid extensive luminal compromise on one plane.

Endoscopy_UCTN_Code_CPL_1AH_2AC 
D. Verma, C. Pham, A. Madan

Division of Gastroenterology, The University of Texas Health Science Center,

Houston, Texas, USA

\section{References}

1 D'Amico G, de Franchis $R$ Cooperative Study Group. Upper digestive bleeding in cirrhosis. Post-therapeutic outcome and prognostic indicators. Hepatology 2003; 38: $599-$ 612

2 Carbonell N, Pauwels A, Serfaty L et al. Improved survival after variceal bleeding in patients with cirrhosis over the past two decades. Hepatology 2004; 40: 652 -659
3 Garcia-Tsao G, Sanyal AJ, Grace ND, Carey W. Prevention and management of gastroesophageal varices and variceal hemorrhage in cirrhosis. Hepatology 2007; 46 $922-938$

4 Lui HF, Stanley AJ, Forrest EH et al. Primary prophylaxis of variceal hemorrhage: a randomized controlled trial comparing band ligation, propranolol, and isosorbide mononitrate. Gastroenterology 2002; 123: 735 744

5 Lo GH, Chen WC, Chen MH et al. Banding ligation versus nadolol and isosorbide mononitrate for the prevention of esophageal variceal rebleeding. Gastroenterology 2002; 123: $728-734$
Bibliography

DOI 10.1055/s-0029-1214856

Endoscopy 2009; 41: E200 - E201

(C) Georg Thieme Verlag KG Stuttgart · New York . ISSN 0013-726X

\section{Corresponding author}

\section{A. Madan, MD, FACP}

Division of Gastroenterology

The University of Texas Health Science Center

6431 Fannin Street, MSB 4.234

Houston

TX 77030

USA

Fax: +1-713-5006699

anandmadan@hotmail.com 